\title{
Prevalencia de agentes enteropatógenos en síndrome diarreico agudo en niños hospitalizados y ambulatorios
}

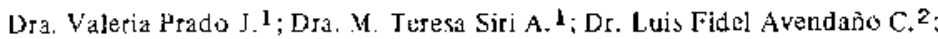 \\ Dra. Stephanie Braun J 3: Dra. F.liana Marambio I..4; Dr, Jván Jorquera B. ${ }^{3}$; \\ T.M. Jucía Reyes M.1: T.M. Juan Martinez D. 1: T.M. Nora Maunani ..1 \\ Prevalence of bacterial and viral pathogens among children \\ with acute diarrhea at hospital and out patient clinics
}

\begin{abstract}
In order to evaluate the relative importance of bacterial and yiral enteropathogens assocjated with acute diarrhea in childrem (at hospital and jn out patients visics) we developed an etiologic study along two summer periods. The first summer period (1983) included 102 patients with diarrea ( 58 children taken from hospital wards and 44 from out patient clinics) and 66 conltuls. In the second summer piriod (1986), I 61 children with diarrhea were studied (80 came from hospital and 81 from out patient injics) together with 71 controls matched by age. In patients with diarrhea, the most prevalent agents were enteropathogenic $\mathrm{F}$. colj (1:PLC) (37\%), enterotoxigenic l:. coli (LTEC) $(21 \%)$, rotavirus $(16 \%)$ and shigella $(12 \%$. In the control group LPJ:C. I:TEC, campylobacter and rotavirus were found at significantly lower trecuencies $115 \% \quad 19 \% \quad 1.5 \%$ o\% $0 \%$ respectively). Among patients requiring admission for their diarrheal diseas. the frecuency of EPEC and rotavirus was significantly higher in comparison with ambulatory patients $\{45$ vs $26 \%$ and 24 us $9 \%$ correspondingly)
\end{abstract}

(Key words: acute diarrhea, diarrheal disease, ethiology).

La diarrea aguda es un padecimiento frecuente en lactantes y pre-escolares en áreas en desarrolio y nuestro país no escapa a esta realidad. Diferentes estudios prospectivos han determinado, quc en los primeros 5 años de vida los niños que habitan en países latinoamericanos, asiáticos $\theta$ africanos sufren como promedio 2 a 4 episodios de diarrea por año, observándose la mayor incidencia en los niños menores de 12 meses ${ }^{1-5}$. En los últimos años con el respaldo de instituciones como la Organización Mundial de la Salud y UNICEF, se han desplegado muchos esfuerzos a nivel mundial para tratar de disminuir la mortalidad y morbilidad por diarrea y como resultado se han logrado avances sustanciales en determinar los agentes causales, sus mecanismos de patogenicidad y los trastornos fisiopatológicos que caracterizan al síndrome diarreico agudo. Este nuevo conocimiento ha permitido el desarrollo de vacunas contra algunos agentes enteropatóge-

1. Unidad microbiologia, Depto. Salud Pública, Divisiôn Ciencias Médicas Oriente, Facultad de Mədicina, Universidad de Chile.

2. Unidad virologia, Depto. Microbiología y Para sitología, División Ciencias Médjcas Norte, Facultad de Medicina, Universidad de Chile.

3. Becado programa especialista microbiología.

4. Instituto de Salud Pública de Chije. nos como E. coli enterotoxigénicos, shigella, rotavirus ${ }^{6-3}$, cuya utilidad esta en estudio.

Pata definir las estrategias preventivas más ütiles en cada comunidad es indispensable iden. tificar la importancia relativa que tienen los diferentes agentes enteropatógenos descritos.

Nuestro interés estuvo dirigido a determinar comparativamente la prevalencia de enteropatógenos bacterianos y virales en un grupo de lactantes hospitalizados por diarrea aguda con deshidratación moderada a severa, en relación a un grupo de lactantes que acuden por diarrea aguda al consultorio y se tratan en forma arrbulatoria, en un estudio prospectivo durante 2 períodos estivales, para defìnir cuáles agentes son responsables de los casos más severos. La presente comunicación corresponde a los resultados obtenidos durante los períodos: noviembre 1982 a marzo 1983 y noviembre 1985 a marzo 1986.

\section{MATERIAL Y METODOS}

Para los propósitos de este estudio se trabajó con la siguiente definición de diarrea: cuadro de comienzo brusco, con evacuación de 3 o más deposiciones diarias. de mayor contenido acuoso de lo normal, con o sin dementos patológicos como mucus, pus $y$, o, sangte; con una duración mayor de un día yenor de 7 .

Si consideraron niños menores de 2 años de estrato socio-económico bajo con diarrea aguda y controles que 
no habian recibido antimicrobianos durante los 7 dias previos de ingreso al estudin, en los siguientes perjudos:

Verano 83: Se incluycron 168 pacientes: 58 hospitalizados por diurses aguda en el Hospital Luis Calvo Muckenna. 44 que consul taron por diafrea en Consultorio La Faena (Area Metropolitana Oriente) y 66 niños controles, de consulta ambulatorio.

Vorano 86: Sᄂ цstudiaron 232 lactantes: 80 pitiontes con diarrea hospitalizados en el misne centro asistential, 8J que consultaron ambulaturiamente $y$ 7l nirjos controles. Los controles fueron pareados por tad y estrato solio-económico y fueron stleccionados intre los niños que acudían a control de niño sano al centro de salud de lat Fuena y algunos 22 esan niños hospitalizados pur palología diferente a diarrea aguda.

Las muestras de deposiclanes (! por cade nit̄o) fueron recolectadas del parial de leces recién smitidiss o mediante hicopado rectal, sc uransportaron en medio Cary Blair y se sembraron dentro de 4 a 6 horas. Seguin el grupo de estudio, las muestras se recoltictaron el primer día de hospitalización o en al momento de consulta.

\section{Procesamiento é identificaction de enteropatógenos}

Cada muestra de deposjción se sejnbró en agar SS. atgar Mac Conkey, agar CampybaP y tampón fosfato satino $\mathrm{pH} 7,2$; se agregó agar sangre cor ampicilina en el segundo período de estudio.

la identificación de silmonclla y shigella, se ctectuó de acuerdo a los criterios establecidos por tedwards $y$ Ewing 14

l.a pesquisa de Yersinia enterocolítica se hizo de acuerdo a pautas establccidas por OMS 15

Para el estudio de eanpylobacter se utilizó ayar Campy-BAt: agar Brucella base adiciomado de $5 \%$ de sangre de cordero $y$ de antibiciticos como inhibidures de la flora agregada (vancomicini - trimetoprim - polimixina $B$ - anfotericina $y$ cefalotina). Se incubó en jarra Gas Pak sin catalizador por $4 B$ horas a $42{ }^{\circ} \mathrm{C}$. $A$ las colonias características, grises, brillantes, se les cstudió la Inorfologia por Gran, producción de oxictasa $y$ er talasa. Posteriormente sc observó crecimiento a temperturas de $42^{\circ} \mathrm{C}, 370^{\circ} \mathrm{C}$ y $25^{\circ} \mathrm{C}$, para diferenciar Campylobacter jejuni y Canpylobacter coli y prueba de TTC, siguitendo recomendaciones de OMS 15 . La producción de betalactamasa en las cepas de campylobacter se determinó por la técnica de la cefalosporina cromógena ; nituoctsina 16

Para el aislamiento de Atromonas hydrophila se utilizó una placa de agar sangre base adicionado de $5 \%$ de sangre de cordero y $15 \mu \mathrm{g} / \mathrm{ml}$ de ampicilina 17 .

La típilicación de E. coli enteropatógeno siguió da siguiente secuencia: Lus serogrupos clásicos fueron identificados serológicamente con antisueros OK (Dif co) $018-020-025-026-055-011$ I - $0119-0125$ - 0127 - 0128 - 0142, mediante reacción de aglutinación en lámina y posteriormente el serogrupa fue confirmado por el Instituto de Salud Pública (SP) de Chile, mediante titulación del antígeno somático $\mathrm{O}$. Los $\mathrm{F}$. coli invasures fueron identificados a partir de cepas con biotipos compatibles incapaces de decarboxilar la lisina e inmovilos en las cuales se efectuó prueba de Serény 18 $y$, posteriormentc, serotipificación con antisueros correspondientes a serogrupos invasores: $028-029-$ 032 - 042 - 044 - 0112 - 0124 - 0136 - .0143 0152 - 0156 (suministrados por Dr. Luiz Trabulsi, Escola Paulista de Medicina, Sao Paulo, Brasij). En todas ayuellas cepas de $E$. coli no pertenecientes a las 2 wategorias anteriores se determinó la capacidad de producir enterotoxinas ( $\boldsymbol{E}$. coli toxigénicos) por los siguientes métodos: La detección de toxina LT se efectuó mediante la técnjé de inmunoprecipitación de Biken, descrita por Ilonda 19,20, que consiste básicamente en haces crecer las colonias de E. coli en un medio sólido (agar Biken) adicionado de lincomicina en coneentración de $90 \mathrm{mcg} / \mathrm{ml}$ y tenfrentarlas a un suero anti LT; empleanos un suero anti-cólers fabricado por nosotros inoculando conejos albinos con toxoide de cólera. En atda placa siempre sc incluyer on como cepas controles E. coli 40T productora de tuxina LT $y$ t. coli $\mathrm{K} 12$ tokina LT negativa (suministradas por Dr, L. Trabulsi). Mediante este método se estudiaron 709 cepas de E. coli. Como técnica de referencia se utilizaron líneas celulures VEkO $y$ CHO en las cuales la toxina LT produce alteraciones morfológicas características 21,22,23. Para inocular las diferentes líneas celulares, se cultivó una colección de cepas de cada nirjo en medio de Evans 24 (1 a 7 copas por paciente) en agitación tontinua durante 18 horas a $37^{\circ} \mathrm{C}$ y postetiormente se ptucedió a centrifugar a 3.500 Ipm durante 30 minutos. Sc obtuvieron de este modo 172 sobrenadantes de culectiones de 5 . coli correspondientes a igual número de pacientes que fueron mantenidos a $-30^{\circ} \mathrm{C}$ hasta su uso: cn los restantes pacientes no se aislason cepas de I: coli diferentes à TECt:P clásicos o jnvasores. Con estos subrenadantes se inocularon microplacas plásticas (LIMBRO) en las cuales previamente se logró obterner monocapas de células VFRO y CHO (se scmbraron aproximadanente $2 \times 10^{4}$ cólulas por cada pocillo) inoculat ndo $25 \mu$ de sobrenadante de cultivo de É. coli. Los efectos sobre las células fueron observados con jecturas a las 6 y $18 \mathrm{~h}$ utilizando un microscopio invertido; se consideró cono positivo la prescncia de alteraciones norfológicas en una proporción igual o superior a $75 \%$ de las vélulas.

Cuando la lectura no tue concluyente se prepararon sobrenadantes por separado con cada una de las cepas incluidas en la colección: esto ocurrió con 58 coleccio nes, proctediéndose a preparar 297 nuevos sobrenadantes con las cepas correspondientes.

lin el segundo poriodo de estudio, se investigó producción de toxina LT mediante LiLISA 25 y paralelamente se utilizaron técnicas de ingeniería genética para identificar los genes que codifican toxina LT mediante hibridización de DNA con sondas marcadas radioactivamente, preparadas en el Centro de Desarrollo de Vacunas de ta Universidad de Maryland.

Lit toxina ST fue detectada mediante inoculación intragástrica en ratones lact:urtes, según la técnica descrita originalmente por Dean 25 , pero en este estudio se inoculó igualmente sobrenadante de una colvcción de cepas de cada pacienti ( 1 a 7 cepas) ${ }^{26}$. Adomás se utilizaron sondas genéticas con plasmidias de STh $y$ STp.

La investigación de rotavirus se efectuó mediante la técnica de electrofotesis del RNA viral en geles de politucrilamida ${ }^{27}$, en las muestras obtenidas en el verano 85-86.

\section{RESULTADOS}

La tabla 1 muestra la proporción comparativa con que se encontraron asociados los diferentes enteropatógenos estudiados, en casos de diarrea aguda o infecciones asintomáticas, en ambos períodos de verano.

En el grupo de niños con diarrea, los principales agentes detectados fueron $E$. coli enteropató. geno (ECEP) como primera frecuencia $(37,3 \%$ y 
Tabla I.

Frecuencia de bacterías erteropatógenas y rotavirus aislados en lactantes con diafrea $y$ controles en 2 periodos de verano

\begin{tabular}{|c|c|c|c|c|c|c|c|c|c|c|}
\hline \multirow{3}{*}{ Enteropatógeno } & \multicolumn{5}{|c|}{ VERANO $19 B 3$} & \multicolumn{4}{|c|}{ VERANO 1986} & \multirow{3}{*}{$p^{*}$} \\
\hline & \multicolumn{2}{|c|}{$\begin{array}{l}\text { Grupo Diarrea } \\
n=102\end{array}$} & \multicolumn{2}{|c|}{$\begin{array}{c}\text { Grupo Cantrol } \\
n=66 \\
\end{array}$} & \multirow[t]{2}{*}{$\mathrm{P}^{*}$} & \multicolumn{2}{|c|}{$\begin{array}{c}\text { Grupo Diarrea } \\
n=162\end{array}$} & \multicolumn{2}{|c|}{$\begin{array}{c}\text { Grupo Control } \\
\mathrm{n}=71 \\
\end{array}$} & \\
\hline & $\mathrm{NO}$ & $\%$ & 10 & $\%$ & & No & $\%$ & No & $\%$ & \\
\hline E. coli enteropatógerro & 38 & 37.3 & 10 & 15,2 & 0,001 & 57 & 34,4 & 11 & 15,5 & 0,002 \\
\hline E. coli enterotoxigénico & 22 & 21,6 & 13 & 19,7 & NS & 28 & 17,4 & 6 & 8.5 & 0,07 \\
\hline E. coli enteroinvasor & 2 & 2,0 & 1 & 1,5 & NS & 3 & 1,9 & 1 & 1,4 & NS \\
\hline Shigeila & 13 & 12,7 & 0 & - & 0,002 & 14 & 8,7 & 0 & - & 0,01 \\
\hline Campylobactet & 8 & 7,8 & 1 & 1,5 & 0,07 & 9 & 5,6 & 0 & . & 0,04 \\
\hline Salmonella & 4 & 3,9 & 0 & . & NS & 1 & 0,6 & 0 & - & NS \\
\hline Yersinia & 0 &.- & 0 & - & & 0 & $\therefore$ & 0 & - & \\
\hline Aeromonas & ni & - & $n \grave{i}$ & $\rightarrow$ & & 0 & - & 0 & - & \\
\hline Rotavirus 1 & $\mathrm{ni}$ & - & ni & -. & & 26 & 16.1 & 2 & 2,8 & 0,004 \\
\hline Asociaciones & 24 & 23,5 & 3 & 4,5 & 0,001 & 18 & 11,2 & 1 & 1,4 & 0,01 \\
\hline Negativo & 36 & 35,3 & 44 & 66,7 & 0,00006 & 59 & 36,6 & 54 & 76,0 & 0,00000003 \\
\hline
\end{tabular}

${ }^{1}$ Rotavirus se estudió en 144 pacientes y 71 controles en periodo 86. ni $=$ no investigado.$$
* x^{2}
$$

Tabla 2.

lonportancia relativa de agentes enteropatógenos bacterianos y virales en lactantes con diarrea aguda, a nivel hospitalario y ambulatorio

\begin{tabular}{|c|c|c|c|c|c|c|c|c|c|c|}
\hline \multirow{3}{*}{ l:ntex opatógeno } & \multicolumn{4}{|c|}{ VFRANO 83} & \multirow{3}{*}{$P$} & \multicolumn{4}{|c|}{ VERANO 86} & \multirow{3}{*}{$P$} \\
\hline & \multicolumn{2}{|c|}{$\begin{array}{c}\text { Hospital } \\
n=58\end{array}$} & \multicolumn{2}{|c|}{$\begin{array}{l}\text { Consultorio } \\
\mathrm{n}=44\end{array}$} & & \multicolumn{2}{|c|}{$\begin{array}{c}\text { Hospital } \\
n=80\end{array}$} & \multicolumn{2}{|c|}{$\begin{array}{c}\text { Consultorio } \\
n=81\end{array}$} & \\
\hline & No & $\%$ & 80 & $\%$ & & 190 & $\%$ & No & $\%$ & \\
\hline I. cali enteropatógeno & 28 & 48,3 & 10 & 22,7 & 0,008 & 36 & 45,0 & 21 & 25,9 & 0,01 \\
\hline I: coli enterotoxigénico & 12 & 20.7 & 10 & 22,7 & NS & 19 & 23,8 & 9 & $1 \mathbf{1}, 1$ & 0,01 \\
\hline E. coli enteroinvasor & 1 & $1, ?$ & $\mathbf{1}$ & 2,3 & NS & 2 & 2,5 & 1 & 1,2 & NS \\
\hline Shigella & 11 & 19,0 & 2 & 4,5 & 0,03 & 6 & 7.5 & 8 & 9,9 & $\mathrm{ks}$ \\
\hline Campylobacter & 3 & 5,2 & $\$$ & 11,4 & NS & 3 & 3,7 & 6 & 7,4 & xs \\
\hline Salmonella & 3 & 5,2 & 1 & 2,3 & NS & 1 & 1.2 & 0 & 0 & \\
\hline Yersinia & 0 & 0 & 0 & 0 & & 0 & 0 & 0 & 0 & \\
\hline Aesomonas & ni & & ni & & & 0 & 0 & 0 & 0 & \\
\hline Ratayirusl & $\mathrm{ni}$ & & nj & & & 19 & 23.7 & 7 & 8,6 & 0,009 \\
\hline Negativo & 14 & 24,1 & 22 & 50 & 0,006 & 17 & 21,3 & 42 & 51,8 & 0,00006 \\
\hline
\end{tabular}

ni = no investjgado.

$1=$ Rotavirus se investigó en 79 pacjentes hospitalizados y 65 que consultaron en forma ambulatoria.

$35,4 \%$ ), luego E. coli enterotoxigénico (ECET), $21,6 \%$ y $17,4 \%$, enseguida rotavirus $(16,1 \%)$ y higella $(12,7$ y $8,7 \%)$.

En el grupo control se aislaron ECEP, ECET, ECEI, campylobacter y rotavirus, resultando significativamente inferior la frecuencia de ECEP, strigella y rotavirus; respecto a ECET y campylobacter se observó diferencia sólo en el periodo de verano 86 en el cual el número de pacientes estudiados fue superior. No se observaron diferencias en la frecuencia de aislamiento de ECEI $y$ salmonella en ambos períodos (mediante prueba de $\mathrm{Chi}^{2}$ ). No se aistó ninguna cepa de yersinia ni aeromonas.

La diferencia en la frecuencia de recuperación de varios enteropatógenos investigados entre niños con diarrea y asintomáticos fue altamente significativa.

En la tabla 2 se analiza la importancia relativa de los agentes enteropatógenos estudiados en los pacientes con diarrea de diferente grado de severidad. En el grupo de niños que necesitaron hospitalización los principales agentes identifica. dos en los 2 períodos de estudio fueron $E$. coll 
enteropatógeno, E. coli enterotoxigénico, Sitigella y Rotasins: en este grupo de pacientes fue posible identificar uno o más de los agentes investigados en el $76 \%$ o $79 \%$ de los casos, lo cua] resultó significativamente superior a lo observado en el grupo que se atendió en Consultorio.

En los pacientes ambulatorios se logró identificar algún agente enteropatógeno en el $50 \%$ de los casos, siendo los principales agentes detecta. dos E. coli enteropatógeno, E. coli enterotoxigénico, shigella y campylobacter. Este último ocupó el segundo lugar en el periodo de verano 83 y fue desplazado por shigella al tercer lugir en el verano 86. En ambos períodos campylobacter mantuvo una frecuencia relativa de aislamiento supcrior en el grupo de niños ambujatorios sin alcanzar sin embargo significación estadística. En este grupo de niños la frecuencia de ECEP, rotavirus, shigella (año 83) y HCET (año 86) fueron estadisticamente inferiores.

Al analizar la incidencia de los principales agentes asociados a diarrea por grupos de edad. (tabla 3) se abservó que las infecciones por $E$. coli enteropatúgeno tienen un inpacto mayor en lactantes menores de 6 meses $(\mathrm{p}<0,0001)$. E. coli enterotoxigénico y rotavirus afectan en mayor proporción a nit̄onos mayores de 6 meses. no alcanzando valores significativos. Las infecciones por shigella y campylobacter en cambio, aumentan su frecuencia en lactantes mayores de 6 meses en forma significativa $(\boldsymbol{p}=0,03$ y 0,059 respectivamente) según prueba dc análisis de diferencia entre proporciones (Z).

\section{COMENTARIOS}

Si bien nuestro estudio no refleja la participación relativa de agentes enteropatógenos bacterianos y de rotavirus en todos los casos de diarrea que se producen en lactantes de los estratos más pobres en nucstro medio, ya que muchos cuadros diarreicos son tratados por la madre en la casa sin
Llegar a consultar, la información obtenida nos muestra cuáles son los principales agentes que se asocian a los cuadros de diarrea que motivan a la madre a solicitar ayuda médica. En este grupo seleccionado de pacientes, 4 son los agentes de mayor importancia epidemiológica: E. coli enteropatógeno, E. coli toxigénico, rotavirus y shigella (tabla I).

Estos resultados difieren sustancialmente de lo observado en países industrializados, en los cuales rotavirus tiene una incidencia mucho más elevadi, con porcentajes de $35 \%$ en consultas privadas y $47 \%$ en lactantes hospitalizados y las bacterias enteropatógenas se aislan sólo en un $2 \%$ en ambos grupos de pacientes 27 . Esto indicaría yue la frecuencia de infecciones entéricas bacterianas se correlaciona en forma inversa con el nivel de vida $y$ las condiciones de saneamiento básico.

La signiticativa recuperación de uno o más de los agentes investigados en los pacientes con diarra en lorma categórica que en nuestro medio la diarrea aguda infantil es de origen infeccioso.

En estudios efectuados en otros paises del tercer mundo como Bangladesh, con condiciones ambientales quizás peores que las nuestras, E. coli enterotoxigénico, shigella y rotavirus fueron los agentes que sc asociaron al $70 \%$ de los cpisodios de diarrea con deshidratación ${ }^{28}$; lamentablemente en ese estudio no se incluyó la identificación de E. coli enteropatógeno.

En los niños asintomáticos se aistaron pre. dominantemente E. coli enteropatógeno. pero como lo hemos analizado en publicaciones anteriores, dos serogrupos son difesentes y la frecuencia es significativamente inferior. ${ }^{29}$ Llama la atención que la frecuencia de $E$. coli enterotoxigénico fue igual en niños con diarrea y en controles en el verano 83 y diferente en el período 86 . En un estudio en que se caracteri. zaron las cepas toxigénicas, observamos que existe un amplio predominio de cepas que pro.

Tabla 3.

Incidencia por grupo etario de los principales enteropatógenos asociados a diarrea aguda

\begin{tabular}{|c|c|c|c|c|c|c|c|c|c|c|c|c|}
\hline \multirow[b]{2}{*}{ Edadmeses } & \multirow[b]{2}{*}{$\mathrm{NO} 1$} & \multicolumn{2}{|c|}{ EPEC } & \multicolumn{2}{|c|}{ ETEC } & \multicolumn{2}{|c|}{ SHIGI:LLA } & \multicolumn{2}{|c|}{ Campylobacter } & \multicolumn{3}{|c|}{ ROTAVIRUS } \\
\hline & & 100 & $\%$ & $\mathrm{~N}^{\mathrm{O}}$ & $\%$ & $N^{O}$ & $\%$ & No & $\%$ & No1 & $N^{\circ}$ & $\%$ \\
\hline$<6$ & 91 & 52 & 57,1 & 15 & 16,5 & 5 & 5,5 & 3 & 3,3 & 43 & 5 & 11,6 \\
\hline $6-12$ & 94 & 32 & 34 & 22 & 23,4 & 11 & 11,7 & 6 & 6,4 & 60 & 18 & 30 \\
\hline J2-24 & 72 & 11 & 15,3 & 11 & 15,3 & 10 & 13,8 & 8 & 11,1 & 38 & 3 & 7,9 \\
\hline$>24$ & 6 & 0 & - & 2 & - & 1 & & 0 & - & 3 & 0 & - \\
\hline Total & 263 & 95 & - & 50 & - & 27 & & 17 & & 144 & 26 & \\
\hline
\end{tabular}

$N^{\circ} 1=N^{\circ}$ de pacientes estudiados en cada grupo etario. 
ducen enterotoxina termoestable (ST) exclusivamente, expresan Factor de Colonización I (CFAI) y pertenecen al serotipo 0153: $\mathrm{H6}^{30}$; esto estaría indicando que habría una cepa endémica predominante en nuestro ambiente, la cual a través de infecciones repctidas podria inducir una immunidad parcial. Se requieren otras investigaciones para poder entender mejor la epidemiología de las infecciones por E. coli enterotoxigénico en nuestros lactantes. Respecto a los E. coli enteroinvasores tienen baja inciden. cia en la diarrea aguda y también se asociaron a infecciones asintomaticas. La frecuencia de shigella, campylobacter y rotavirus en lactantes asintomáticos fue significativamente inferior a los niños con diarrea.

Al analizar separadamente los agentes identificados en niños hospitalizados, respecto de aquellos atendidos ambulatoriamente (tabla 2) vemos que en el último grupo sc aislaron enteropatógenos cn menor proporción y que los agentes más importantes en los 2 niveles son E. coli enteropatógeno y E. coli enterotoxigénico.

En los dos períodos de estudio, campylobacter se asoció con relativa mayor frecuencia a tasos ambulatorios que a casos severos. Con shigella se observó una situación inversa en el primer período de verano, pero en el segundo periodo la frecuencia fue similar en ambos grupus, reflejando crecmos, fluctuaciones en la epidemiología de la shigellosis, ya que desde el punto de vista metodológico en ambos períodos participó cl mismo equipo de investigadores y se utilizaron las mismas técnicas de laboratorio. En relación a rotavirus su importancia resultó mayor en los casos severos que en cuadros leves que no requicren hospitalizacion, coincidiendo con observaciones de otros investigadores ${ }^{32}$ Llama la atención el relativo alto porcentaje de aisla. miento en niños hospitalizados en verano, en circunstancias que la diarrea por rotavirus es típicamente de invierno. Este hecho se ha encontrado en otros estudios en Chile ${ }^{27}$

No se aisló en ningún paciente cepas de Yersinia enterocclítica ni Aeromonas hydrophila.

Una información de interés que aporta el análisis de estos resultados es la incidencia relativa de los agentes enteropątógenos estudiados por distintos grupos de edad, se observó un cierto patrón en el sentido que las infecciones más precoces serían por E. coli cnteropatógeno, después de los 6 meses cobran mayor importancia rotavirus, shigetla y campylobacter. Sin embargo este patrón observado necesitara ser confirmado, mediante estudios prospectivos de cohortes, ya que este aspecto es primordial que sea claramente establecido para planificar medidas de control específicas como vacunas.
En conclusión, nuestras observaciones han mostrado que en nuestro medio 5 serian los agentes que tienen participación más importante en los casos de diarrea aguda que requieren atención médica y ellos son: $E$. coli enteropa. tógeno, E. coli enterotoxigénico, shigella, campylobacter y rotavirus. Será necesario definir mejor, en futuros estudios, la importancia de E. coli enterotoxigénico. En base a estos antecedentes para disminuir la incidencia de diarrea aguda en lactantes, dentro del conjunto de otras estratcgias debería darse prioridad cuando estén disponibles, a vacunas que otorguen protección frente a estos agentes.

\section{RESUMEN}

Para conocer importancia relativa de agentes cnteropatógenos bacterianos y virales en niños con diarrea aguda que necesitan atención médica de nivel primario o secundario, se efectuó un estudio etiológico durante 2 príodos de verano. Primer período, verano 1983, 102 pacientes que cumplian con definición pre establecida de dia. trea aguda (58 de hospital, 44 de consultorio) y 66 . controles; segundo períudo, verano 86 se estudiaron 161 pacientes con diarrea ( 80 hospitalizados, 81 ambulatorios) y 71 controles pareados por edad. En el grupo con diarre los principales agentes detectados fueron $E$. coli enteropatúgeno (ECEP) (37\%), E. coli enterotoxigénico (ECET) $(21 \%)$, rotavirus (16\%) shigella (12\%) En el grupo control se aislaron ECEP, ECET, campylobacter y rotavirus; la frecuencia de ECEP, shigella y rotavirus fue significativamente inferior. En los pacientes hospitalizados por diarrea, con especto a los ambulatorios la prevalencia de ECEP y rotavirus en hospitalizados fuc significativamente superior. Hubo mayor tenden. cia al aislamiento más frecuente de campylobacter en los pacientes ambulatorios.

\section{AGRADECIMIENTOS}

Estos estudios han sido financiados con fondos de OMS/OPS Grant CDD Progranme $107 \mathrm{CHI} 2$ y del Departamento de Investigación y Biblioteca de la Uni versidad de Chile (DIB) Proyecto No M2104-8623.

Agradecemos la asesoría técnica de la Dra. Karen D'Ottone y 'T.M. Sra. Nancy Rivera F., del Instituto de Salud Pública, por la confirmación de la serotipificación de E. coli y al Dr. Myron Levine, Direcior del Centro de Desarrollo de Vacunas de la Universidad de Maryland U.S.A., la confirmación de 1 ;. coli enteropatógenos y E. coli enterotoxigénicos A la Dra. Catterine Ferreccio, académico de la Facultad de Medicina, Universidad de Chile, debemos agradecer en análisis estadístico de los resultados. 


\section{REFERENCIAS}

1. Snyder, $J_{-}$., Merson, M.H.: The magnitude of the gobal problem of acute diarthocal disease: a review of active surveillance data. Bull WHO 40:65, 1982.

2. Mata. L.J.: The children of Santa María Cauqué a prospective field study of heallh and growth. Cambridge, Man MIT Press 1978.

3. Newell, $K . W$ : Diarheal disease of infancy in C.ali Colombia: study design and summary report on isolated disease agents Bull of P.4HO 10: 143 . 1976.

4. Lie, K.J. Diarrhoea among infants in a crowded area of Djakarta, Indonesia: a longitudinal study from birth to two years. Bull WHO 34: 197, 1966.

5. Black, R.E.,Brow'n, K.H, Becker, S. et al. Longitudinal studjes of infectious diseases and physical growth of childen in rural Bangladesh II. Incidence and etiology of diarrhea. Am J lipidemil 115: 315 , 1982 .

6. Letine, M.M., Kaper, JB., Black, R.E. et of: New knowledge on pathogenesis of bacterial enteric infections as applied to vaccine development. Microbiol Rev 47: 510 , 1983.

7. Klipstein, R.A., Engert, R.F., Clements, S.D. at al. Vaccine for enterotoxigenic Lscherichia coli based on synthetic hat-stable coxin aross-linked to the B sub unit of heat labile tokin. J Infect Dis 147: 318 , 1983.

8. Evans, D.C;., Graham, D.Y.. Evans, D.G., Jr.: Administration of purifted colonization factor antigens (ClAl, CFAlI) of enterotoxigenic Escherichia coli to yolunteers. Gastroenterology 87: $934,1984$.

9. Schmidt, M., Kelly, E.P., Tseng, L.Y. et al. Towards an oral $I_{i}$. coli piles vaccine for tiavelers'diarthea: susceptibility of purified colonization factor antigen 11 to proteolytic digestion. Gastroenter ology 88 : $1575,1985$.

10. Vesikari, $T$., Isolauri, E. D'Hondt, E, et al: Protection of infants against Rotavirus diarrhea by RIT 4237 attenuated bovine Rotavirus strain vaccine. Lancet i: $977,1984$.

11. Losonsky. G., Rennels, M.B.. Kopikian, A.Z. et al: Safety, infectivity, transmissibility and immunogenicity of Rhesus Rotavirus vaccine (MMU 18006) in infants. Pediatr Infect Dis 5: 25, 1986.

12. Mel, D.M., Arsit., B.L., Radiovanovic. M.L. et al. Live oral Shigella vaccine: vaccination schedule and the effect of booster dose. Acta Microbiol Acad Sci Hung 21: 109, 1974.

13. Levine, M.M., Gangarosa; E.J., Barrow, W.B. et al: Shigellosis in custodial institutions. $V$ effect of intervention with streptomycin-dependent shigella sonnei vaccine in an institution with endemic disease. Am J Epidemiol 104: 88, I976.

14. Edwards, P.R., Ewing, W.H.: Identification of Enterobacteriaceae, Shigella, Capítulo 7. Salmonella, Capitulo 9. $6^{\text {a }}$ Ea. Minneapolis, Minn. Burgess publishing $C_{0 .}, 137$ and 181, 1976.

15. OMS/OPS Documento oftcial $80.283^{\circ}$ Comité Ejecurivo. Programa de Control de Enfermedades Diarreicas.
16. Lennette, E.H., Bolows, A., Hausler, W.J., Truant, P.J.: Manual of Clinical Microbiology. Third tid. Washington D.C. American Society for Microbiolo $\mathrm{gy}, 483,1980$.

17. Von Graeventtz, A., Bucher, C.: Jivaluation of differential : and selective media for jsolation of Aeromonas and Plesiomonas sp from human feces. J Cin Microbiol 17: 16, 1983.

18. Sereny, B.: Гxperimental Shigella Kerátoconjuntisitis: a preliminary report. Atta Microbiol Acad Sci Hung 2: 293, 1955 .

19. Honda, T., Iaga. S., Takeda, Y.. Mirwatani, T.: Moditied l:lek Test for detection of heallabile enterotoxin of enterotoxiganic Escherichía coli. J Clin Microbiol 13: 1, 1981.

20. Honda, $T$, Glass, R., Akhtar, Q. Kibriva, A.: A sinple assay to ditect $E$. coli producing LT enterotoxin: results of a field study of the Biken test in Валgladesh. Lancat 1: 609, 1981.

21. Konowolchuek, J., Speirs, J.I: Response of various cell lines to Eschertichia coli toxic products. 'Can J Microb 25: 335, 1979.

22. Speirs, Srartick; S., Konowalchuck: Assay of Escherichia colf heat-labile enterotoxin witl Vera cells. Infect Immun 16: 617, 1977 .

23. Giverront, R.L., Brunton, L.L., Schoitman, T.C.. Rebhun, L.L., Gilman, A.G.:' Cyclit Adenosine Monophusphate and alteration of chineso hataster ovary cell morphology: a rapid, sensitive in vitro assay for the enterotoxins, of vibrio cholcrac and Escherjchia coli. Infect Immun 10:320, 1974.

24. Evans, D.G., Evans, D.J. Pierce, N.: Diferences in the response of ratiit small intestine to $L . T$ and $S T$ enterotoxins of E. coli. Infect Immun $7: 873,1973$.

25. Dean, A.G., Ching, Y., Ch, Williams, R.G. et al. Test for $L$. coli entcrotoxin using nufant nice: application in study of diarthes in children on Honolulu. J Infect Dis 125: 40?, 1972.

26. Merson, M.H., Sack, R.G., Kihriya, A.K.M.G. et al The use of colony pools for diagnosis of enterotoxingenic Lscherichia coli diarthea. J Clin Microbiol 9: 493,1979 .

27. Avendaño, L.F., Sponcer, E., Calderón, A., Martínez, $A_{\text {.: }}$ Infección por rotavirus en lactantes con diarrey aguda. Rev Med Clije 1 I 1: 240, 1983.

28. Koopmen, J.S., Turkish, V.J., Monto, A.S. er at: Patterns and etiology of diarriea in three clinical set tings. Am J lipidemiol 1 19: 114, 1984.

29. Black, R.E, Merson, M.H.; Huq. I. et al: Incidence and severity of Rotavitus and Jischerichia coli diarrhea in rural Bangladesh. Lancet i: $141,1981$.

30. Prado, Y., Broun, S., Bosh, P. el al: Análisis de E. coli enteropatógenos clásicos como causa endémica de diarrea aguda en nin̄os chìenos. Rev Clil Pediatr. 55: 171,1984

31. Aguero. M.E., Reyes, L., Prado, V. et al: Enterotoxigenic Escherictia coli is a population of infants with diarrhea in Chile. J Clin Microbiol 22: 576 . 1985 .

32. Black, R.E., Merson, M.H., Rahman, S.M.M., Yunus $M$, et al: A two year study of bacterial, viral and parasitic agents associnted with diarrhea in rutal Bangladesh. J Infect Dis 142: 660, 1980. 